\title{
Comparison of claudin-3 and claudin- 4 expression in bilateral and unilateral breast cancer
}

\author{
Jolanta SZADE ${ }^{1, \star}$, Michał KUNC ${ }^{1}$, Beata PIECZYŃSKA-UZIĘBŁO ${ }^{1}$, Maciej ŚWIERBLEWSKI ${ }^{2}$, Wojciech BIERNAT ${ }^{1}$, Jacek JASSEM ${ }^{3}$, Elżbieta SENKUS ${ }^{3}$ \\ ${ }^{1}$ Department of Pathomorphology, Medical University of Gdansk, Gdansk, Poland; ${ }^{2}$ Department of Surgical Oncology, Medical University of \\ Gdansk, Gdansk, Poland; ${ }^{3}$ Department of Oncology and Radiotherapy, Medical University of Gdansk, Gdansk, Poland
}

${ }^{*}$ Correspondence: jszade@gumed.edu.pl

Received August 16, 2020 / Accepted October 14, 2020

\begin{abstract}
Claudins are essential components of tight junctions, which are frequently deregulated in breast cancer. The aim of the current study was to assess claudin-3 and -4 expression in bilateral breast cancer (BBC) and unilateral breast cancer (UBC). Immunohistochemical expression of claudin-3 and claudin-4 was evaluated in tissue microarrays containing 174 cases of BBCs paired with 174 cases of solitary tumors. Each case was classified as claudin-high or claudin-low depending on the $\mathrm{H}$-score value. The results were correlated with histopathological features and the expression of basic breast cancer biomarkers. Median H-scores for claudin-3 were significantly higher in the synchronous BBC (sBBC) than in UBC. Claudin4-high cases were more prevalent than within the whole BBC group, and sBBC and metachronous BBC (mBBC) alone. In the BBC group negative ER, high Ki-67 and high claudin-3 were independent factors correlated with high claudin-4. In the UBC group, Ki-67 $>14 \%$ and high claudin-3 were associated with high claudin- 4 . Our study demonstrates that the expression of claudin-4 is significantly higher in UBC compared to BBC tumors. We also demonstrated that high claudin-4 expression in $\mathrm{BBC}$ is associated with a more aggressive phenotype (lack of steroid receptors, HER2 overexpression, and high $\mathrm{Ki}-67)$. It is possible that claudins down- and upregulation may depend on different triggers and lead to various consequences in UBC and BBC.
\end{abstract}

Key words: claudin-3, claudin-4, immunohistochemical expression, bilateral breast cancer, unilateral breast cancer

Breast cancer affects paired organ in the human body, thus sometimes presents bilaterally. In the vast majority of cases, such phenomenon results from independent clonal malignant proliferations, and not from metastatic spread of ipsilateral tumor [1]. Risk factors for bilateral breast cancer (BBC) include early age at diagnosis, family history of cancer, lobular histology, BRCA1 or BRCA2 mutation, and CHEK2 mutation [2]. Bilateral presentation occurs either synchronously ( $30 \%$ of cases) or metachronously ( $\sim 70 \%$ of cases) [3]. Information about the time of development of contralateral breast tumor is relevant since synchronous (sBBC) and metachronous (mBBC) breast cancers have slightly different biology and prognosis. In our previous works, we showed that $\mathrm{mBBC}$ is characterized by a more aggressive phenotype, expressed by lower expression of estrogen receptor, and stronger expression of cytokeratin 5/6, vimentin, p21, and cyclin B [4-6].

Claudins are essential components of tight junctions, probably serving as their backbone. They show the tetraspan transmembrane structure and possess two extracellular loops (ECL1 and ECL2), which participate in interactions with other components of tight junctions and may serve as receptors [7]. The intracellular segments of claudins are sites of protein binding (e.g. PDZ class 1 of scaffolding proteins), as well as post-translational modifications changing their properties [8]. Homo- or heteromeric combinations of claudin-family members form intracellular strands to promote cell-to-cell adhesion and to control paracellular epithelial permeability [9]. The loss of these properties in neoplastic cells may facilitate invasion and metastatic spread. Indeed, the elements of tight junctions have been found to be abnormally expressed in various human malignancies. Amongst the claudin family of proteins, expression of claudin-1, claudin-3, claudin-4, and claudin-7 seem to be the most frequently altered in cancer, including breast, colon, gastric, hepatocellular, ovarian, pancreatic, prostate, and thyroid carcinomas [10]. The function of claudins in both normal and neoplastic tissues is dynamically regulated, since their phosphorylation may decrease tight junctions' strength [11]. 
To date, there have been no studies investigating claudins expression in BBC. The aim of the present study is to analyze claudin- 3 and claudin- 4 expression in the BBC cohort and control group consisting of unilateral breast cancer (UBC) patients.



Figure 1. Representative immunohistochemical staining for claudin-3 and claudin-4 in breast tumors. A) H\&E staining, B) high claudin-3 membrane and cytoplasmic expression, C) high claudin-4 membrane and cytoplasmic staining

\section{Materials and methods}

BBC samples. 174 specimens of BBCs were collected retrospectively from four Polish institutions. They were compared with 174 cases of solitary tumors selected from the database of the Department of Pathology, Medical University of Gdansk, and matched by age at diagnosis of breast cancer ( \pm 1 year). The detailed information about the cohorts was presented earlier [4-6]. Available formalin-fixed paraffinembedded tissue blocks from breast tumors were collected and centrally verified for diagnosis of invasive breast cancer and for the presence of sufficient invasive tumor to prepare tissue microarrays. All specimens were reviewed for histology according to the 2019 World Health Organization (WHO) classification. The following BBC criteria were used: the presence of in situ lesions, different histological features of both tumors, a higher degree of differentiation of the second tumor, and no evidence of regional or distant metastases from the first tumor. The study was approved by the Ethics Committee of the Medical University of Gdansk, Poland.

Immunohistochemistry (IHC). Immunohistochemistry (IHC) was performed on tissue microarrays (TMA) built using Manual Tissue Microarrayer 1 (Beecher Instr. Inc, Sun Prairie, WI, USA), using 2 representative cores for each tumor. Sections were cut from paraffin blocks at $4 \mu \mathrm{m}$ and stained according to standard procedures, as described by the manufacturers. Incubation with primary antibody claudin-3 (Thermo Scientific RB-9251-P1) and claudin-4 (Thermo Scientific RB-9266-P1) was conducted for $90 \mathrm{~min}$. The Novolink Polymer Detection System (Leica Microsystems $\mathrm{GmbH}$, Wetzlar, Germany) was used for all procedures, apart from the primary antibody and buffers used for antigen retrieval (DAKO, Glostrup).

IHC analysis. IHC scoring was performed by a single experienced pathologist (JSz). The ER and PgR were scored according to Allred (with percentage and intensity scores noted separately) and HER2-according to ASCO/CAP guidelines. For Ki-67, the proportion of positive cells was grouped into three categories: $\leq 14 \%, 15-30 \%$, and $>30 \%$. The proportion of cells stained for CK5/6, vimentin, and EGFR was categorized into negative $(<1 \%) ; 1-10 \%$; and $>10 \%$. E-cadherin expression was considered positive if strong staining was present in $\geq 75 \%$ of cells. For centrally determined HER2 (2+) cases, the FISH analysis was not routinely performed, but for some patients, the result was available in patients' records. These results were used only for the determination of the surrogate intrinsic tumor phenotype. The surrogate intrinsic phenotype was based on the expression of ER, HER2, and Ki-67. Claudin-3 and -4 expression was assessed utilizing $\mathrm{H}$-score, which combines the percentage of positively stained cells $(0-100)$ and staining intensity $(0-3)$. The obtained multiplied score ranged between $0-300$ (Figure 1).

Statistics. Associations between categorical variables were evaluated using the $\chi^{2}$ test. Odds ratios (OR) were calculated 
with 95\% confidence intervals (95\% CI) using logistic regression. Similarly, in multivariate analysis, logistic regression was used (stepwise backwards logistic regression, 95\%). The Mann-Whitney test was performed to compare the median $\mathrm{H}$-scores of claudin-3 and claudin-4 between groups (BBC, $\mathrm{sBBC}, \mathrm{mBBC}, \mathrm{UBC})$. In the multivariate analysis, only cases with no missing data were included. Statistical significance was assumed when $\mathrm{p}<0.05$. Calculations were performed using Statistica software (Statsoft Co, USA, version 13), licensed to the Medical University of Gdansk.

\section{Results}

We classified breast cancer samples according to their median $\mathrm{H}$-score values in the entire cohort $(\mathrm{BBC}+\mathrm{UBC})$ for each claudin, assigning them as claudin-low or claudinhigh cases. The cut-off value for claudin-3 and claudin- 4 high cases was $>80$, and $>140$ respectively. The number of tumors classified as expressing high- or low-claudin did not differ between groups. (Table 1), however, compared to sBBC there was a trend for a higher number of claudin-3 low cases in the UBC group. In fact, median $\mathrm{H}$-scores for claudin-3 compared by the Mann-Whitney test were significantly higher in the sBBC than in the UBC group (Figure 2). Cases designated as claudin-4-high were more prevalent in UBC groups than within the whole BBC group, and sBBC and $\mathrm{mBBC}$ alone. Similarly, the median claudin-4 H-score was significantly higher in the UBC group. sBBC and $\mathrm{mBBC}$ groups demonstrated similar number of both claudin-3 (low/ high) and claudin-4 (low/high) cases. BRCA1 carrier status was determined for 72 patients diagnosed with BBC, and 19 of them had a mutation. A comparison of median claudins $\mathrm{H}$-scores between BRCA1 mutation carriers and sporadic cases revealed no significant differences ( $p=0.143$, the MannWhitney test).

The mBBC group had significantly more claudin-3 negative cases than $\mathrm{sBBC}\left(\mathrm{p}=0.0339, \chi^{2}\right)$. Loss of claudin-3 expression was more frequent in ER-negative subgroup of $\mathrm{BBC}\left(\mathrm{p}=0.025, \chi^{2}\right)$. No significant differences regarding the number of claudin- 4 negative cases were found.

In the BBC group, claudin- 4 was the only independent factor predicting claudin-3 expression (Table 2), whereas negative ER, high Ki-67 were independent factors associated with high claudin-4 (Table 3 ). On the other hand, in UBCs grade 3 and high claudin- 4 independently predicted high claudin-3 expression (Table 4), whereas Ki-67 >14\% was associated with high claudin-4 (Table 5).

\section{Discussion}

In the current study, we demonstrated significant differences in claudin-3 and claudin-4 expression between UBC and $\mathrm{BBC}$. These findings emphasize the distinct biology of bilateral tumors. Moreover, high claudin-4 was associated with a more aggressive phenotype in BBC. Thus, further studies should investigate the potential role of claudins as prognostic factors and therapeutic targets.

Emerging evidence suggests that breast cancers showing downregulated claudins exemplify a distinct intrinsic subgroup called "claudin-low breast cancer", representing approximately $6 \%$ of all breast malignancies [12]. It is

Table 1. Comparison of claudin-3 and claudin- 4 expression in bilateral (BBC) and unilateral breast cancer (UBC) - $\chi^{2}$ test.

\begin{tabular}{lcccccccc}
\hline & \multicolumn{3}{c}{ Claudin-3 } & & \multicolumn{4}{c}{ Claudin-4 } \\
\cline { 2 - 3 } \cline { 6 - 8 } & Low & High & p-value $\left(\chi^{2}\right)$ & & Low & High & p-value $\left(\boldsymbol{\chi}^{\mathbf{2}}\right)$ \\
\hline BBC & 80 & 78 & 0.243 & & 93 & 72 & $<\mathbf{0 . 0 0 1}$ \\
UBC & 97 & 73 & & & 31 & 140 & \\
sBBC & 24 & 31 & 0.199 & & 37 & 20 & 0.108 \\
mBBC & 56 & 47 & & & 56 & 52 & \\
mBBC & 56 & 47 & 0.664 & & 56 & 52 & $<\mathbf{0 . 0 0 1}$ \\
UBC & 97 & 73 & & & 31 & 140 & \\
\hline
\end{tabular}
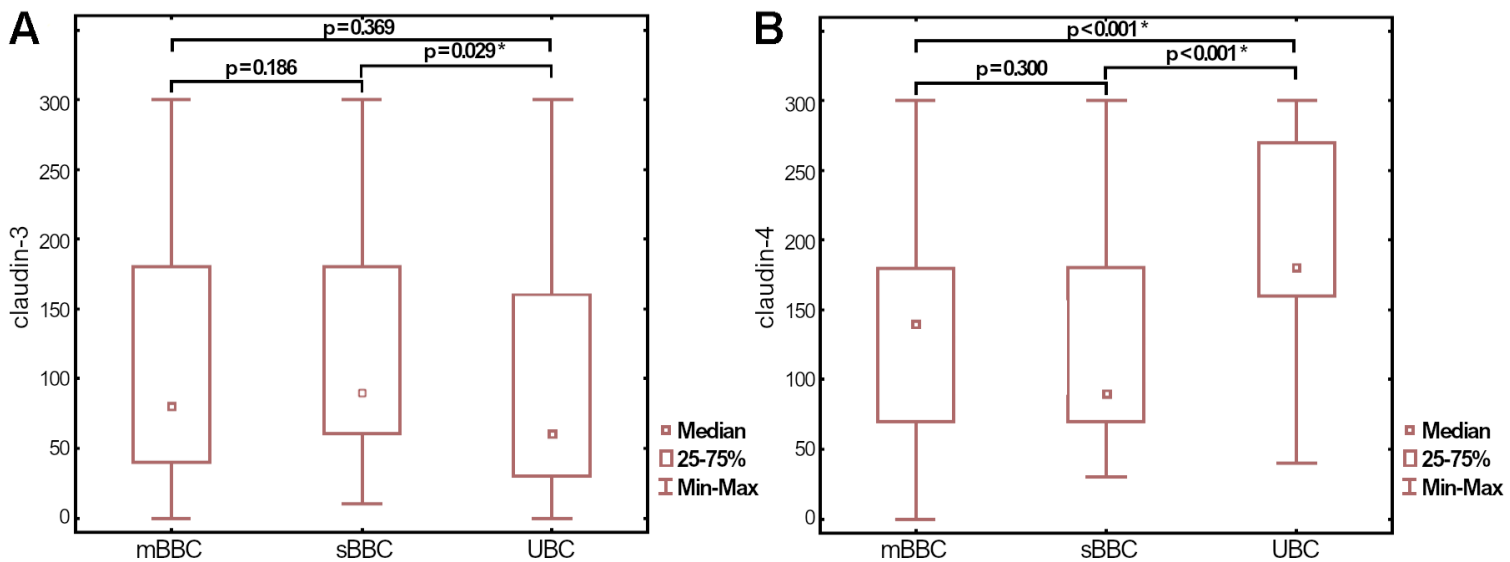

Figure 2. Comparison of median H-scores for claudin-3 (A) and claudin-4 (B) regarding the group. ${ }^{*}$ Significant differences p $<0.05$. Abbreviations: mBBC - metachronous bilateral breast cancer; sBBC - synchronous bilateral breast cancer; UBC - unilateral breast cancer 
Table 2. Factors associated with high claudin-3 in bilateral breast cancer: univariate and multivariate analysis (logistic regression).

\begin{tabular}{|c|c|c|c|c|c|c|}
\hline \multirow{2}{*}{ Factor } & \multicolumn{2}{|c|}{ Claudin-3 } & \multicolumn{2}{|c|}{ Univariate analysis } & \multicolumn{2}{|c|}{ Multivariate analysis } \\
\hline & Low & High & OR $(95 \% \mathrm{CI})$ & p-value & OR (95\% CI) & p-value \\
\hline Grade $1+2$ vs. 3 & $\begin{array}{l}52(65.0 \%) \\
28(35.0 \%)\end{array}$ & $\begin{array}{l}50(64.1 \%) \\
28(35.9 \%)\end{array}$ & $\begin{array}{c}0.962 \\
(0.501-1.846)\end{array}$ & 0.906 & & \\
\hline ER negative vs. positive & $\begin{array}{l}18(23.1 \%) \\
60(76.9 \%)\end{array}$ & $\begin{array}{l}14(17.9 \%) \\
64(82.1 \%)\end{array}$ & $\begin{array}{c}0.729 \\
(0.334-1.594)\end{array}$ & 0.429 & & \\
\hline PR negative vs. positive & $\begin{array}{l}33(41.3 \%) \\
47(58.8 \%)\end{array}$ & $\begin{array}{l}28(35.9 \%) \\
50(64.1 \%)\end{array}$ & $\begin{array}{c}0.798 \\
(0.420-1.516)\end{array}$ & 0.490 & & \\
\hline HER2 $0-1$ vs. $2-3$ & $\begin{array}{l}65(81.3 \%) \\
15(18.8 \%)\end{array}$ & $\begin{array}{l}53(67.9 \%) \\
25(32.1 \%)\end{array}$ & $\begin{array}{c}0.489 \\
(0.234-1.021)\end{array}$ & 0.057 & & \\
\hline Ki- $67<14 \%$ vs. $>14 \%$ & $\begin{array}{l}23(28.7 \%) \\
57(71.3 \%)\end{array}$ & $\begin{array}{l}19(24.7 \%) \\
58(75.3 \%)\end{array}$ & $\begin{array}{c}0.812 \\
(0.399-1.650)\end{array}$ & 0.565 & & \\
\hline CK5/6 negative vs. positive & $\begin{array}{l}50(63.3 \%) \\
29(36.7 \%)\end{array}$ & $\begin{array}{l}56(72.7 \%) \\
21(27.3 \%)\end{array}$ & $\begin{array}{c}0.647 \\
(0.328-1.275)\end{array}$ & 0.208 & & \\
\hline Vimentin $<1 \%$ vs. $>1 \%$ & $\begin{array}{l}49(62.0 \%) \\
30(38.0 \%)\end{array}$ & $\begin{array}{l}56(73.7 \%) \\
20(26.3 \%)\end{array}$ & $\begin{array}{c}1.714 \\
(0.865-3.396)\end{array}$ & 0.122 & & \\
\hline Claudin-4 low vs. high & $\begin{array}{l}79(45.9 \%) \\
93(54.1 \%)\end{array}$ & $\begin{array}{c}38(25.3 \%) \\
112(74.7 \%)\end{array}$ & $\begin{array}{c}0.399 \\
(0.248-0.642)\end{array}$ & $<0.001$ & $\begin{array}{c}0.327 \\
(0.165-0.647)\end{array}$ & 0.001 \\
\hline E-cadherin negative vs. positive & $\begin{array}{c}7(8.8 \%) \\
73(91.3 \%)\end{array}$ & $\begin{array}{c}2(2.6 \%) \\
74(97.4 \%)\end{array}$ & $\begin{array}{c}0.282 \\
(0.057-1.402)\end{array}$ & 0.122 & & \\
\hline Age $<50$ vs. $>=50$ & $\begin{array}{l}34(42.5 \%) \\
46(57.5 \%)\end{array}$ & $\begin{array}{l}22(28.2 \%) \\
56(71.8 \%)\end{array}$ & $\begin{array}{c}0.532 \\
(0.274-1.032)\end{array}$ & 0.062 & & \\
\hline EGFR $<1 \%$ vs. $>1 \%$ & $\begin{array}{l}58(73.4 \%) \\
21(26.6 \%)\end{array}$ & $\begin{array}{l}55(71.4 \%) \\
22(28.6 \%)\end{array}$ & $\begin{array}{c}0.905 \\
(0.448-1.827)\end{array}$ & 0.781 & & \\
\hline sBBC vs. mBBC & $\begin{array}{l}56(70.0 \%) \\
24(30.0 \%)\end{array}$ & $\begin{array}{l}47(60.3 \%) \\
31(39.7 \%)\end{array}$ & $\begin{array}{c}0.650 \\
(0.336-1.256)\end{array}$ & 0.200 & & \\
\hline
\end{tabular}

Table 3. Factors associated with high claudin-4 in bilateral breast cancer: univariate and multivariate analysis (logistic regression).

\begin{tabular}{|c|c|c|c|c|c|c|}
\hline \multirow{2}{*}{ Factor } & \multicolumn{2}{|c|}{ Claudin-4 } & \multicolumn{2}{|c|}{ Univariate analysis } & \multicolumn{2}{|c|}{ Multivariate analysis } \\
\hline & Low & High & OR $(95 \% \mathrm{CI})$ & p-value & OR $(95 \% \mathrm{CI})$ & p-value \\
\hline Grade $1+2$ vs. 3 & $\begin{array}{l}65(69.9 \%) \\
28(30.1 \%)\end{array}$ & $\begin{array}{l}41(56.9 \%) \\
31(43.1 \%)\end{array}$ & $\begin{array}{c}0.570 \\
(0.299-1.084)\end{array}$ & 0.087 & & \\
\hline ER negative vs. positive & $\begin{array}{l}13(14.1 \%) \\
79(85.9 \%)\end{array}$ & $\begin{array}{l}22(31.0 \%) \\
49(69.0 \%)\end{array}$ & $\begin{array}{c}2.728 \\
(1.260-5.909)\end{array}$ & 0.011 & $\begin{array}{c}3.071 \\
(1.278-7.380)\end{array}$ & 0.012 \\
\hline PR negative vs. positive & $\begin{array}{l}27(29.0 \%) \\
66(71.0 \%)\end{array}$ & $\begin{array}{l}34(47.9 \%) \\
37(52.1 \%)\end{array}$ & $\begin{array}{c}2.246 \\
(1.177-4.286)\end{array}$ & 0.014 & & \\
\hline HER2 $0-1$ vs. $2-3$ & $\begin{array}{l}76(81.7 \%) \\
17(18.3 \%)\end{array}$ & $\begin{array}{l}49(68.1 \%) \\
23(31.9 \%)\end{array}$ & $\begin{array}{c}0.477 \\
(0.231-0.981)\end{array}$ & 0.044 & & \\
\hline Ki- $67<14 \%$ vs. $>14 \%$ & $\begin{array}{l}33(35.5 \%) \\
60(64.5 \%)\end{array}$ & $\begin{array}{l}11(15.5 \%) \\
60(84.5 \%)\end{array}$ & $\begin{array}{c}0.333 \\
(0.154-0.720)\end{array}$ & 0.005 & $\begin{array}{c}0.338 \\
(0.143-0.798)\end{array}$ & 0.013 \\
\hline CK5/6 negative vs. positive & $\begin{array}{l}64(63.3 \%) \\
29(36.7 \%)\end{array}$ & $\begin{array}{l}45(64.3 \%) \\
25(35.7 \%)\end{array}$ & $\begin{array}{c}0.731 \\
(0.375-1.427)\end{array}$ & 0.359 & & \\
\hline Vimentin $<1 \%$ vs. $>1 \%$ & $\begin{array}{l}62(68.1 \%) \\
29(31.9 \%)\end{array}$ & $\begin{array}{l}45(65.2 \%) \\
24(34.8 \%)\end{array}$ & $\begin{array}{c}0.877 \\
(0.452-1.702)\end{array}$ & 0.698 & & \\
\hline Claudin-3 low vs. high & $\begin{array}{l}79(67.5 \%) \\
38(32.5 \%)\end{array}$ & $\begin{array}{c}93(45.4 \%) \\
112(53.6 \%)\end{array}$ & $\begin{array}{c}0.399 \\
(0.248-0.642)\end{array}$ & 0.000 & $\begin{array}{c}0.332 \\
(0.161-0.685)\end{array}$ & 0.003 \\
\hline E-cadherin negative vs. positive & $\begin{array}{l}12(13.0 \%) \\
80(87.0 \%)\end{array}$ & $\begin{array}{c}1(1.4 \%) \\
69(98.6 \%)\end{array}$ & $\begin{array}{c}0.097 \\
(0.012-0.762)\end{array}$ & 0.027 & & \\
\hline Age $<50$ vs. $>=50$ & $\begin{array}{l}39(41.9 \%) \\
54(58.1 \%)\end{array}$ & $\begin{array}{l}20(27.8 \%) \\
52(72.2 \%)\end{array}$ & $\begin{array}{c}0.533 \\
(0.275-1.130)\end{array}$ & 0.061 & & \\
\hline EGFR $<1 \%$ vs. $>1 \%$ & $\begin{array}{l}71(77.2 \%) \\
21(22.8 \%)\end{array}$ & $\begin{array}{l}46(65.7 \%) \\
24(34.3 \%)\end{array}$ & $\begin{array}{c}0.567 \\
(0.283-1.134)\end{array}$ & 0.109 & & \\
\hline sBBC vs. mBBC & $\begin{array}{l}56(60.2 \%) \\
37(39.8 \%)\end{array}$ & $\begin{array}{l}52(72.2 \%) \\
20(27.8 \%)\end{array}$ & $\begin{array}{c}1.718 \\
(0.886-3.331)\end{array}$ & 0.109 & & \\
\hline
\end{tabular}


Table 4. Factors associated with high claudin-3 in unilateral breast cancer: univariate and multivariate analysis (logistic regression).

\begin{tabular}{|c|c|c|c|c|c|c|}
\hline \multirow{2}{*}{ Factor } & \multicolumn{2}{|c|}{ Claudin-3 } & \multicolumn{2}{|c|}{ Univariate analysis } & \multicolumn{2}{|c|}{ Multivariate analysis } \\
\hline & Low & High & OR $(95 \% \mathrm{CI})$ & p-value & OR $(95 \% \mathrm{CI})$ & p-value \\
\hline Grade $1+2$ vs. 3 & $\begin{array}{l}69(71.1 \%) \\
28(28.9 \%)\end{array}$ & $\begin{array}{l}35(47.9 \%) \\
38(52.1 \%)\end{array}$ & $\begin{array}{c}0.374 \\
(0.198-0.706)\end{array}$ & 0.002 & $\begin{array}{c}0.398 \\
(0.202-0.785)\end{array}$ & 0.008 \\
\hline ER negative vs. positive & $\begin{array}{l}18(18.6 \%) \\
79(81.4 \%)\end{array}$ & $\begin{array}{l}21(28.8 \%) \\
52(71.2 \%)\end{array}$ & $\begin{array}{c}1.772 \\
(0.862-3.642)\end{array}$ & 0.110 & & \\
\hline PR negative vs. positive & $\begin{array}{l}21(21.6 \%) \\
76(78.4 \%)\end{array}$ & $\begin{array}{l}27(37.0 \%) \\
46(63.0 \%)\end{array}$ & $\begin{array}{c}2.124 \\
(1.079-4.183)\end{array}$ & 0.029 & & \\
\hline HER2 $0-1$ vs. $2-3$ & $\begin{array}{l}61(62.9 \%) \\
36(37.1 \%)\end{array}$ & $\begin{array}{l}38(52.1 \%) \\
35(47.9 \%)\end{array}$ & $\begin{array}{c}0.641 \\
(0.346-1.187)\end{array}$ & 0.157 & & \\
\hline Ki- $67<14 \%$ vs. $>14 \%$ & $\begin{array}{l}32(33.7 \%) \\
63(66.3 \%)\end{array}$ & $\begin{array}{l}16(21.9 \%) \\
57(78.1 \%)\end{array}$ & $\begin{array}{c}0.553 \\
(0.275-1.112)\end{array}$ & 0.096 & & \\
\hline CK5/6 negative vs. positive & $\begin{array}{l}70(75.3 \%) \\
23(24.7 \%)\end{array}$ & $\begin{array}{l}50(69.4 \%) \\
22(30.6 \%)\end{array}$ & $\begin{array}{c}0.747 \\
(0.375-1.486)\end{array}$ & 0.405 & & \\
\hline Vimentin $<1 \%$ vs. $>1 \%$ & $\begin{array}{l}77(81.1 \%) \\
18(18.9 \%)\end{array}$ & $\begin{array}{l}43(59.7 \%) \\
29(40.3 \%)\end{array}$ & $\begin{array}{c}0.357 \\
(0.173-0.696)\end{array}$ & 0.003 & & \\
\hline Claudin-4 low vs. high & $\begin{array}{l}25(26.6 \%) \\
69(73.4 \%)\end{array}$ & $\begin{array}{c}5(6.8 \%) \\
68(93.2 \%)\end{array}$ & $\begin{array}{c}0.203 \\
(0.073-0.561)\end{array}$ & 0.002 & $\begin{array}{c}0.260 \\
(0.089-0.756)\end{array}$ & 0.013 \\
\hline E-cadherin negative vs. positive & $\begin{array}{l}13(13.4 \%) \\
84(86.6 \%)\end{array}$ & $\begin{array}{c}1(1.4 \%) \\
72(98.6 \%)\end{array}$ & $\begin{array}{c}0.09 \\
(0.011-0.703)\end{array}$ & 0.022 & & \\
\hline Age $<50$ vs. $>=50$ & $\begin{array}{l}34(35.1 \%) \\
63(64.9 \%)\end{array}$ & $\begin{array}{l}27(37.0 \%) \\
46(63.0 \%)\end{array}$ & $\begin{array}{c}1.088 \\
(0.578-2.047)\end{array}$ & 0.795 & & \\
\hline EGFR $<1 \%$ vs. $>1 \%$ & $\begin{array}{l}73(75.3 \%) \\
24(24.7 \%)\end{array}$ & $\begin{array}{l}48(65.8 \%) \\
25(34.2 \%)\end{array}$ & $\begin{array}{c}1.584 \\
(0.812-3.090)\end{array}$ & 0.177 & & \\
\hline
\end{tabular}

Table 5. Factors associated with high claudin-4 in unilateral breast cancer: univariate and multivariate analysis (logistic regression).

\begin{tabular}{|c|c|c|c|c|c|c|}
\hline \multirow{2}{*}{ Factor } & \multicolumn{2}{|c|}{ Claudin-4 } & \multicolumn{2}{|c|}{ Univariate analysis } & \multicolumn{2}{|c|}{ Multivariate analysis } \\
\hline & Low & High & OR (95\% CI) & p-value & OR $(95 \% \mathrm{CI})$ & p-value \\
\hline Grade $1+2$ vs. 3 & $\begin{array}{l}21(67.7 \%) \\
10(32.3 \%)\end{array}$ & $\begin{array}{l}84(60.0 \%) \\
56(40.0 \%)\end{array}$ & $\begin{array}{c}0.714 \\
(0.313-1.631)\end{array}$ & 0.424 & & \\
\hline ER negative vs. positive & $\begin{array}{c}6(19.4 \%) \\
25(80.6 \%)\end{array}$ & $\begin{array}{c}33(23.6 \%) \\
107(86.4 \%)\end{array}$ & $\begin{array}{c}1.285 \\
(0.486-3.399)\end{array}$ & 0.613 & & \\
\hline PR negative vs. positive & $\begin{array}{c}7(22.6 \%) \\
24(77.4 \%)\end{array}$ & $\begin{array}{l}41(29.3 \%) \\
99(80.7 \%)\end{array}$ & $\begin{array}{c}1.420 \\
(0.567-3.553)\end{array}$ & 0.454 & & \\
\hline HER2 $0-1$ vs. $2-3$ & $\begin{array}{l}21(67.7 \%) \\
10(32.3 \%)\end{array}$ & $\begin{array}{l}79(56.4 \%) \\
61(43.6 \%)\end{array}$ & $\begin{array}{c}0.617 \\
(0.271-1.406)\end{array}$ & 0.250 & & \\
\hline Ki-67 <14\% vs. $>14 \%$ & $\begin{array}{l}15(50 \%) \\
15(50 \%)\end{array}$ & $\begin{array}{c}33(23.7 \%) \\
106(76.3 \%)\end{array}$ & $\begin{array}{c}0.311 \\
(0.138-0.704)\end{array}$ & 0.005 & $\begin{array}{c}0.195 \\
(0.058-0.649)\end{array}$ & 0.008 \\
\hline CK5/6 negative vs. positive & $\begin{array}{c}22(78.6 \%) \\
6(21.4 \%)\end{array}$ & $\begin{array}{l}99(72.3 \%) \\
38(27.7 \%)\end{array}$ & $\begin{array}{c}0.711 \\
(0.267-1.888)\end{array}$ & 0.493 & & \\
\hline Vimentin $<1 \%$ vs. $>1 \%$ & $\begin{array}{c}27(90 \%) \\
3(10 \%)\end{array}$ & $\begin{array}{l}94(67.6 \%) \\
45(32.4 \%)\end{array}$ & $\begin{array}{c}0.232 \\
(0.067-0.806)\end{array}$ & 0.021 & & \\
\hline Claudin-3 low vs. high & $\begin{array}{c}25(83.3 \%) \\
5(16.7 \%)\end{array}$ & $\begin{array}{l}69(50.4 \%) \\
68(49.6 \%)\end{array}$ & $\begin{array}{c}0.203 \\
(0.073-0.561)\end{array}$ & 0.002 & $\begin{array}{c}0.245 \\
(0.082-0.727)\end{array}$ & 0.011 \\
\hline E-cadherin negative vs. positive & $\begin{array}{c}7(22.6 \%) \\
24(77.4 \%)\end{array}$ & $\begin{array}{c}7(5.0 \%) \\
133(95.0 \%)\end{array}$ & $\begin{array}{c}0.180 \\
(0.058-0.561)\end{array}$ & 0.003 & & \\
\hline Age $<50$ vs. $>=50$ & $\begin{array}{l}9(29.0 \%) \\
22(71 \%)\end{array}$ & $\begin{array}{l}52(37.1 \%) \\
88(62.9 \%)\end{array}$ & $\begin{array}{c}1.444 \\
(0,619-3,372)\end{array}$ & 0.395 & & \\
\hline EGFR $<1 \%$ vs. $>1 \%$ & $\begin{array}{c}23(74.2 \%) \\
8(25.8 \%)\end{array}$ & $\begin{array}{l}97(69.3 \%) \\
43(30.7 \%)\end{array}$ & $\begin{array}{c}0.785 \\
(0.325-1.894)\end{array}$ & 0.589 & & \\
\hline
\end{tabular}


associated with poor prognosis and probably with resistance to certain chemotherapeutic agents. This is consistent with the widely accepted hypothesis that in cancer the disruption of tight junctions leads to increased cellular motility, and subsequent loss of cohesion, invasion, and finally the formation of metastases. However, these proteins may be either down- or upregulated in cancer and many studies reported claudin-3 and claudin-4 overexpression in breast cancer $[13,14]$. Claudin- 4 is especially upregulated in metastases, reflecting the more aggressive behavior of such tumors [15]. In the face of the biological function of claudins, this phenomenon looks paradoxical, but probably claudins upregulation leads to disruption of tight junction integrity, similarly to their loss. In cancers arising in the background of the BRCA1 mutation (which is a risk factor for $\mathrm{BBC}$ ), there is a high expression of claudin-1, claudin-3, and claudin-4 $[16,17]$. Interestingly, neoplastic cells expressing claudin-3 and -4 may be targeted by clostridium perfringens enterotoxin (CPE) [18]. CPE selectively binds to these claudins and then induces the disintegration of the cellular membrane and subsequent cytolysis. Clostridial toxin is now extensively evaluated as a potential therapeutic agent for tumors overexpressing claudin-3 and claudin- 4 .

The study by Lanigan et al. conducted on invasive breast cancer samples showed that increased claudin- 4 expression was associated with worse survival, high tumor grade, HER2 expression, and negative ER [13]. Similar results were obtained in the cohort composed of ER-negative breast cancers, as claudin- 1 and -4, but not claudin-3, were correlated with basal features (expression of Her-2, EGFR, and CK5/6) [19]. Both claudin-3 and claudin-4 showed a correlation with Ki-67 in that study. Most of these findings are consistent with our BBC cohorts, whereas amongst UBC cases no clear associations between high claudins expression with basal immunophenotype were found. Importantly, in our study complete loss of claudin-3 was found to be more common in ER-negative tumors, suggesting that either the overexpression or a loss of tight junction elements may increase the aggressiveness of breast cancer. In the UBC group, there was a positive association between claudin-3 and -4 with E-cadherin expression on univariate, but not multivariate analysis. It is not surprising, since the knockdown of either CLND3 or CLDN4 gene results in downregulation of E-cadherin [20].

We observed a significant positive correlation between claudin-3 and claudin- 4 expression. The genes encoding both these proteins are located in the nearby loci on chromosome 7 and probably their expression is co-regulated [21]. In fact, co-expression of claudin-3 and claudin- 4 has been observed in various normal and neoplastic tissues [22]. They form heteromeric complexes when expressed in the same cell but are unable to interact heterotypically (head-to-head) unless some amino acid substitutions in extracellular loops are made [23]. Nevertheless, the exact role of these interactions in human malignancies is yet to be elucidated.
BRCA1-related cancers frequently show the overexpression of claudins, including -3 and -4 [24]. However, we did not observe any difference between BRCA-1 associated BBC and sporadic $\mathrm{BBC}$ regarding the expression of evaluated claudins. Possibly, it results from the fact that whereas claudin- 1 and -6 are independent predictors of BRCA- 1 status, claudin- $3,-4$, and -7 are rather dependent on the ER status [24].

The role of claudin- 3 and claudin- 4 in breast cancer may depend on the general phenotype of a tumor. In triplenegative cancers, the claudin- 4 expression seems to be favorable, whereas in luminal cancers-unfavorable prognostic factor [25].

In our study, the $\mathrm{H}$-score cut-off value between the high- and low expression of claudin-4 was relatively high. Most authors reported a lower number of claudin- 4 overexpressing tumors, but Soini et al. demonstrated strongly positive claudin- 4 staining in $72 \%$ of cases [26]. These discrepancies probably result from the use of various antibodies during immunohistochemistry and different scoring of staining. In conclusion, our study demonstrates that the expression of claudin- 4 is significantly higher in $\mathrm{UBC}$ cases compared to BBC tumors. We also demonstrated that a high claudin-4 expression in BBC is characterized by a more aggressive phenotype: lack of steroid hormone receptors, HER2 overexpression, and high Ki-67. These findings indicate that high levels of claudin- 4 in bilateral tumors correlate with a more aggressive phenotype. On the opposite, the claudin-3 high phenotype was correlated with grade 3 and PgR negativity in UBC. Thus, it is possible that claudins down- and upregulation may depend on different triggers and lead to various consequences in solitary and bilateral tumors.

Acknowledgments: This work was financially supported by the Polish Ministry of Science and Higher Education (grant no. NN 407 206435) and was partly supported by institutional grants of Medical University of Gdańsk, Poland (02-0095/07/267).

\section{References}

[1] IMYANITOV EN, HANSON KP. Molecular pathogenesis of bilateral breast cancer. Cancer Lett 2003; 191: 1-7. https:// doi.org/10.1016/s0304-3835(02)00523-2

[2] NAROD SA. Bilateral breast cancers. Nat Rev Clin Oncol 2014; 11: 157-166. https://doi.org/10.1038/nrclinonc.2014.3

[3] TAKAHASHI H, WATANABE K, TAKAHASHI M, TAGUCHI K, SASAKI F et al. The impact of bilateral breast cancer on the prognosis of breast cancer: a comparative study with unilateral breast cancer. Breast Cancer 2005; 12: 196-202. https://doi.org/10.2325/jbcs.12.196

[4] SENKUS E, SZADE J, PIECZYŃSKA B, ZACZEK A, ŚWIERBLEWSKI $\mathrm{M}$ et al. Are bilateral breast cancers and breast cancers coexisting with ovarian cancer different from solitary tumors? A pair-matched immunohistochemical analysis aimed at intrinsic tumor phenotype. Pathol Int 2014; 64: 508-517. https://doi.org/10.1111/pin.12202 
[5] SENKUS E, SZADE J, PIECZYNSSKA B, ZACZEK A, PIKIEL J et al. Are synchronous and metachronous bilateral breast cancers different? An immunohistochemical analysis aimed at intrinsic tumor phenotype. Int J Clin Exp Pathol 2014; 7: 353-363.

[6] SENKUS E, SZADE J, PIECZYŃSKA B, KUNC M, PLISZKA A et al. Are synchronous and metachronous bilateral breast cancers different? An immunohistochemical analysis focused on cell cycle regulation. Histol Histopathol 2018; 33: 55-64. https://doi.org/10.14670/HH-11-887

[7] KRAUSE G, WINKLER L, MUELLER SL, HASELOFF RF, PIONTEK J et al. Structure and function of claudins. Biochim Biophys Acta 2008; 1778: 631-645. https://doi. org/10.1016/j.bbamem.2007.10.018

[8] KWON MJ. Emerging roles of claudins in human cancer. Int J Mol Sci 2013; 14: 18148-18180. https://doi.org/10.3390/ ijms140918148

[9] VAN ITALLIE CM, ANDERSON JM. Claudin interactions in and out of the tight junction. Tissue Barriers 2013; 1 : e25247. https://doi.org/10.4161/tisb.25247

[10] MORIN PJ. Claudin proteins in human cancer: promising new targets for diagnosis and therapy. Cancer Res 2005; 65: 9603-9606. https://doi.org/10.1158/0008-5472.CAN-052782

[11] FINDLEY MK, KOVAL M. Regulation and roles for claudin-family tight junction proteins. IUBMB Life 2009; 61: 431-437. https://doi.org/10.1002/iub.175

[12] SABATIER R, FINETTI P, GUILLE A, ADELAIDE J, CHAFFANET $M$ et al. Claudin-low breast cancers: clinical, pathological, molecular and prognostic characterization. Mol Cancer 2014; 13: 228. https://doi.org/10.1186/14764598-13-228

[13] LANIGAN F, MCKIERNAN E, BRENNAN DJ, HEGARTY S, MILLIKAN RC et al. Increased claudin-4 expression is associated with poor prognosis and high tumour grade in breast cancer. Int J Cancer 2009; 124: 2088-2097. https://doi. org/10.1002/ijc.24159

[14] SKÁlOVÁ H, HÁJKOVÁ N, MAJEROVÁ B, BÁRTU゚ M, POVÝŠIL $\mathrm{C}$ et al. Impact of chemotherapy on the expression of claudins and cadherins in invasive breast cancer. Exp Ther Med 2019; 18: 3014-3024. https://doi.org/10.3892/ etm.2019.7930

[15] JIWA LS, VAN DIEST PJ, HOEFNAGEL LD, WESSELING J, WESSELING P et al. Upregulation of Claudin-4, CAIX and GLUT-1 in distant breast cancer metastases. BMC Cancer 2014; 14: 864. https://doi.org/10.1186/1471-2407-14-864
[16] MADARAS L, BALINT N, GYORFFY B, TOKES AM, BARSHACK I et al. BRCA Mutation-Related and ClaudinLow Breast Cancer: Blood Relatives or Stepsisters. Pathobiology 2016; 83: 1-12. https://doi.org/10.1159/000439135

[17] DANZINGER S, TAN YY, RUDAS M, KASTNER MT, WEINGARTSHOFER $S$ et al. Association of Cytokeratin 5 and Claudin 3 expression with BRCA1 and BRCA2 germline mutations in women with early breast cancer. BMC Cancer 2019; 19: 695. https://doi.org/10.1186/s12885-019-5908-6

[18] WALTHER W, PETKOV S, KUVARDINA ON, AUMANN J, KOBELT D et al. Novel Clostridium perfringens enterotoxin suicide gene therapy for selective treatment of claudin-3- and -4-overexpressing tumors. Gene Ther 2012; 19: 494-503. https://doi.org/10.1038/gt.2011.136

[19] BLANCHARD AA, SKLIRIS GP, WATSON PH, MURPHY LC, PENNER C et al. Claudins 1, 3, and 4 protein expression in ER negative breast cancer correlates with markers of the basal phenotype. Virchows Arch 2009; 454: 647-656. https:// doi.org/10.1007/s00428-009-0770-6

[20] GERHARD R, RICARDO S, ALBERGARIA A, GOMES $\mathrm{M}$, SILVA AR et al. Immunohistochemical features of claudin-low intrinsic subtype in metaplastic breast carcinomas. Breast 2012; 21: 354-360. https://doi.org/10.1016/j. breast.2012.03.001

[21] LAL-NAG M, MORIN PJ. The claudins. Genome Biol 2009; 10: 235. https://doi.org/10.1186/gb-2009-10-8-235

[22] HEWITT KJ, AGARWAL R, MORIN PJ. The claudin gene family: expression in normal and neoplastic tissues. BMC Cancer 2006; 6: 186. https://doi.org/10.1186/1471-2407-6186

[23] DAUGHERTY BL, WARD C, SMITH T, RITZENTHALER JD, KOVAL M. Regulation of heterotypic claudin compatibility. J Biol Chem 2007; 282: 30005-30013. https://doi. org/10.1074/jbc.M703547200

[24] HEERMA VAN VOSS MR, VAN DIEST PJ, SMOLDERS YH, BART J, VAN DER WALL E et al. Distinct claudin expression characterizes BRCA1-related breast cancer. Histopathology 2014; 65: 814-827. https://doi.org/10.1111/ his. 12490

[25] KOLOKYTHA P, YIANNOU P, KERAMOPOULOS D, KOLOKYTHAS A, NONNI A et al. Claudin-3 and claudin-4: distinct prognostic significance in triple-negative and luminal breast cancer. Appl Immunohistochem Mol Morphol 2014; 22: 125-131. https://doi.org/10.1097/ PAI.0b013e31828d9d62

[26] SOINI Y. Claudins 2, 3, 4, and 5 in Paget's disease and breast carcinoma. Hum Pathol 2004; 35: 1531-1536. https://doi. org/10.1016/j.humpath.2004.09.015 